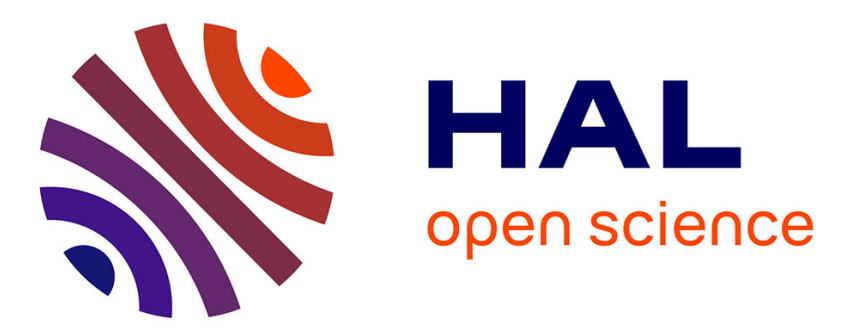

\title{
Non-parametric material state field extraction from full field measurements
}

\author{
Adrien Leygue, Rian Seghir, Julien Réthoré, Michel Coret, Erwan Verron, \\ Laurent Stainier
}

\section{- To cite this version:}

Adrien Leygue, Rian Seghir, Julien Réthoré, Michel Coret, Erwan Verron, et al.. Non-parametric material state field extraction from full field measurements. Computational Mechanics, 2019, 10.1007/s00466-019-01725-z . hal-02158197

\section{HAL Id: hal-02158197 \\ https://hal.science/hal-02158197}

Submitted on 18 Jun 2019

HAL is a multi-disciplinary open access archive for the deposit and dissemination of scientific research documents, whether they are published or not. The documents may come from teaching and research institutions in France or abroad, or from public or private research centers.
L'archive ouverte pluridisciplinaire HAL, est destinée au dépôt et à la diffusion de documents scientifiques de niveau recherche, publiés ou non, émanant des établissements d'enseignement et de recherche français ou étrangers, des laboratoires publics ou privés. 


\title{
Non-parametric material state field extraction
}

\section{from full field measurements}

\author{
Adrien Leygue, Rian Seghir, Julien Réthoré, Michel Coret, \\ Erwan Verron, Laurent Stainier \\ Research Institute in Civil Engineering and Mechanics (GeM) \\ Centrale Nantes, CNRS, UMR 6183 \\ 1 rue de la Noë 44321 Nantes, France.
}

Received: date / Revised version: date

\begin{abstract}
Data-driven computations propose a completely new paradigm to the computational mechanics research community and to experimentalists. Classically, admissible material states can only be obtained experimentally for homogeneous stress/strain configurations or using a parametric optimization of material laws based on heterogeneous tests. Data-driven algorithms aim at circumvent these limitations. However, data-driven algorithms require a large database of admissible material states, otherwise extrapolation is required and some limitations of the classical constitutive equation based approach remain. In this paper, an inverse data-driven approach based on full field measurements is presented. The main idea is to
\end{abstract}


extract, with no assumption on the constitutive equations, rich (i.e. heterogeneous and multiaxial) material state fields from displacement fields and external load measurements. The capability of the proposed method to extract databases of admissible material states and to evaluate stress fields without parametric constitutive equations is illustrated through three examples dedicated to non-linear elasticity, plasticity and dynamics.

\section{Introduction}

Solution algorithms in computational mechanics make use of constitutive equations. They can be purely phenomenological or thermo-dynamically consistent. However, it is unavoidable that, at some point, one has to write down a constitutive equation with some parameters to be determined experimentally. The most common practice is to perform a sufficient number of experiments using samples and loading conditions that lead to homogeneous stress and strain states, within a gauge zone, that can be estimated directly from macroscopic force and displacement measurements. As a consequence, the phase space (stress, strain) that is of high dimension is only explored along a few radial trajectories. Invoking the symmetry class of the material (isotropy, orthotropy,...), the dimensionality of the phase space can be reduced but at least the invariants of stress and strain tensors remain. The sparse nature of the experimental exploration of the phase space makes the use of the elaborated constitutive equations for predicting the behavior of complex structures a continuous extrapolation of the experimental results. 
Since the early 80's, full-field displacement measurements, like Digital Image Correlation (DIC), have been developed (see [1] for a review). They offer the opportunity for the experimentalists to obtain the full displacement field at the surface of a given specimen. This provides for rich data as the evolution of the strain field can be easily derived and thus, experiments leading to complex heterogeneous material state fields can be analyzed. Actually only the strain field can be measured but not the stress one, which is not that useful for the purpose of material characterization. However, coupling these full field measurements with numerical simulations offers the opportunity to thoroughly compare numerical predictions for a given constitutive law and full-field experimental data. Even more interesting, trying to reduce the scatter between experimental and numerical results, it is possible to adjust the parameters (Finite Element Model Updating FEMU) of the constitutive equations being part of the simulations [2]. This kind of approach has been shown to be extremely robust and reliable $[3,4]$ even in the case of non-linear and history dependent constitutive models. The interested reader should refer to [5] or [6] for an overview of parameter identification using full-field measurements. However, within this framework, one has to use a constitutive equation which parameters are to be identified. Usually this equation is the same as the one developed from the homogeneous experiments but classically, identification based on macroscopic or field data will lead to different parameters. Consequently, the intrinsic limitations of the constitutive equation itself remain unchanged but the parameters are 
more adapted to the considered heterogeneous experiments (but less for the homogeneous ones).

Recently, Kirchdoerfer and Ortiz [7] proposed a data-driven computational mechanics (DDCM) approach. The constitutive equation is replaced by the minimization of the distance, e.g. an energy norm, between the computed state (stress, strain) and a cloud of admissible material states that are supposed to be obtained experimentally. There is thus no need to write down explicitly the constitutive equation. This strategy offers the opportunity to circumvent the limitations of the classical approach described in the previous paragraphs. However, if the experimental data used within this framework for predicting the response of complex structures are still obtained from homogeneous state experiments then the issue regarding the extrapolation of the experimental data remains.

In this paper, an inverse data driven approach is presented. The main idea is to formulate the inverse problem associated to data-driven computational mechanics in order to use the displacement field and load measurements, to recover a set of admissible material states with no assumption on the constitutive equation. The approach can be used on complex geometries and thus the limitations of the classical approach regarding either the homogeneous state experiments or the constitutive equation itself are circumvented. In the next Section, the formulation of the proposed DataDriven Identification (DDI) method is developed. Then, in Section 3, two applications are presented: they illustrate the capability of the proposed 
methodology. In the first example, the case of a non-linear elastic material under quasi-static loading conditions is presented. Then, a linear elastic material subjected to transient loading is analyzed. Indeed, the only difference, in the proposed framework, between quasi-static and dynamics is that there is a volume force distribution arising from the inertia term in balance equations. The acceleration being experimentally accessed as the second derivative of the displacement, the extension of the proposed framework to transient experiments is straightforward. Last, in Section 4, an alternative formulation of the initial DDI problem formulated in Section 2 is presented. The initial problem is solved assuming that a first guess of the stress field is provided, either from a numerical simulation of the experiment using a constitutive law obtained from homogeneous state experiments or from the aforementioned FEMU technique. Then, only the correction to this first guess is sought, what is shown to be efficiently performed over a reduced domain and using a reduced basis. An example involving data for an elastoplastic material is presented. Compared to[8], all the proposed results are new. Those of Section 3.1 are similar to those in the aforementioned paper but a different setup is used. The methodology presented in Section 4 and the results in Section 3.2 are completely new.

\section{Data-Driven Identification}

In this section, the general framework of DDI is presented. The small strain assumption is considered and the phase space for describing the state of 
the material consists in a linear strain tensor $\boldsymbol{\epsilon}$ and a Cauchy stress tensor $\boldsymbol{\sigma}$, each belonging in a 6-dimensional space (after accounting for their symmetry).

The starting point of DDI is the existence of a large database of strain measurements obtained for example by DIC from images acquired during the experiments. Each of these images and the corresponding displacement fields are called snapshot. The applied load at the corresponding time instant is also measured.

A linearized kinematics, discretized on a finite element mesh in which a given quadrature point $e$ admits an integration weight $w_{e}$, is considered. For snapshot $X$, the following quantities are available:

- nodal displacements $\left(\mathbf{u}_{j}^{X}\right)_{j}$

- finite element geometry and connectivity, encoded through a matrix $\left(\mathbf{B}_{e j}^{X}\right)_{j}$, which computes the mechanical strain $\boldsymbol{\epsilon}_{e}^{X}=\sum_{j} \mathbf{B}_{e j}^{X} \cdot \mathbf{u}_{j}^{X}$ at each quadrature point,

- applied nodal forces $\mathbf{f}_{j}^{X}$.

The aim of the DDI technique is to compute a number $N^{*}$ of material states $\left(\boldsymbol{\epsilon}_{i}^{*}, \boldsymbol{\sigma}_{i}^{*}\right)_{i}$ such that:

- for each snapshot $X$ and quadrature point $e$, it is possible to compute the mechanical state $\boldsymbol{\sigma}_{e}^{X}$ which satisfies mechanical equilibrium,

- for each snapshot $X$, a material state $\left(\boldsymbol{\epsilon}_{s(e, X)}^{*}, \boldsymbol{\sigma}_{s(e, X)}^{*}\right)$ can be assigned to each quadrature point $e$ which is the closest to the mechanical state according to a given energetic norm $\|\cdot\|_{\mathbb{C}_{e}}$. This mapping $(e, X) \longrightarrow$ 
$s(e, X)$ allows to associate each mechanical state $(e, X)$ to a material state in the database.

Following Kirchdoerfer and Ortiz [7], we consider

$$
\left\|\left(\boldsymbol{\epsilon}_{e}, \boldsymbol{\sigma}_{e}\right)\right\|_{\mathbb{C}_{e}}^{2}=\frac{1}{2}\left(\boldsymbol{\epsilon}_{e}: \mathbb{C}_{e}: \boldsymbol{\epsilon}_{e}+\boldsymbol{\sigma}_{e}: \mathbb{C}_{e}^{-1}: \boldsymbol{\sigma}_{e}\right)
$$

where $\mathbb{C}_{e}$ is a (symmetric positive definite) fourth order stiffness tensor. For identifying the material states, the following global minimization problem is:

$$
\text { solution }=\arg \min _{\boldsymbol{\sigma}_{e}^{X}, \boldsymbol{\epsilon}_{i}^{*}, \boldsymbol{\sigma}_{i}^{*}, s} \mathcal{E}\left(\boldsymbol{\sigma}_{e}^{X}, \boldsymbol{\epsilon}_{i}^{*}, \boldsymbol{\sigma}_{i}^{*}, s\right)
$$

with

$$
\mathcal{E}\left(\boldsymbol{\sigma}_{e}^{X}, \boldsymbol{\epsilon}_{i}^{*}, \boldsymbol{\sigma}_{i}^{*}, s\right)=\sum_{X} \sum_{e} w_{e}^{X}\left\|\left(\boldsymbol{\epsilon}_{e}^{X}-\boldsymbol{\epsilon}_{s(e, X)}^{*}, \boldsymbol{\sigma}_{e}^{X}-\boldsymbol{\sigma}_{s(e, X)}^{*}\right)\right\|_{\mathbb{C}_{e}}^{2}
$$

and subjected to the constraint of global equilibrium equations:

$$
\sum_{e} w_{e}^{X} \mathbf{B}_{e j}^{X} \cdot \boldsymbol{\sigma}_{e}^{X}=\mathbf{f}_{j}^{X} \quad \forall X, j .
$$

In these equations, $\sum_{e}$ stands for the sum over all quadrature points $e$ and, $\sum_{X}$ for the sum over all snapshots. All unknowns are real valued except the state mapping $s$ which is discrete (integer valued). For an arbitrary state mapping, the equilibrium constraints Equation (4) are enforced by means of Lagrange multipliers $\boldsymbol{\eta}_{j}^{X}$, leading to the following problem:

$$
\begin{aligned}
& \delta\left(\sum _ { X } \sum _ { e } \left(w_{e}^{X}\left(\boldsymbol{\epsilon}_{e}^{X}-\boldsymbol{\epsilon}_{s(e, X)}^{*}\right): \mathbb{C}_{e}^{X}:\left(\boldsymbol{\epsilon}_{e}^{X}-\boldsymbol{\epsilon}_{s(e, X)}^{*}\right)\right.\right. \\
& +w_{e}^{X}\left(\boldsymbol{\sigma}_{e}^{X}-\boldsymbol{\sigma}_{s(e, X)}^{*}\right): \mathbb{C}_{e}^{X-1}:\left(\boldsymbol{\sigma}_{e}^{X}-\boldsymbol{\sigma}_{s(e, X)}^{*}\right)- \\
& \left.\left.\sum_{j}\left(w_{e}^{X} \mathbf{B}_{e j}^{X^{T}} \cdot \boldsymbol{\sigma}_{e}^{X}-\mathbf{f}_{j}^{X}\right) \cdot \boldsymbol{\eta}_{j}^{X}\right)\right)=0 .
\end{aligned}
$$


Taking all possible variations yields the following set of equations:

$$
\begin{gathered}
\delta \boldsymbol{\epsilon}_{i}^{*} \Rightarrow \sum_{X} \sum_{e \in S_{i}^{X}} w_{e}^{X} \mathbb{C}_{e}^{X}:\left(\boldsymbol{\epsilon}_{e}^{X}-\boldsymbol{\epsilon}_{s(e, X)}^{*}\right)=0 \quad \forall i \\
\delta \boldsymbol{\sigma}_{i}^{*} \Rightarrow \sum_{X} \sum_{e \in S_{i}^{X}} w_{e}^{X}\left(\mathbb{C}_{e}^{X}\right)^{-1}:\left(\boldsymbol{\sigma}_{e}^{X}-\boldsymbol{\sigma}_{s(e, X)}^{*}\right)=0 \quad \forall i \\
\delta \boldsymbol{\sigma}_{e}^{X} \Rightarrow w_{e}^{X} \mathbb{C}_{e}^{X-1}:\left(\boldsymbol{\sigma}_{e}^{X}-\boldsymbol{\sigma}_{s(e, X)}^{*}\right)-\sum_{j} w_{e}^{X} \mathbf{B}_{e j}^{X} \cdot \boldsymbol{\eta}_{j}^{X}=0 \quad \forall e, X \\
\delta \boldsymbol{\eta}_{j}^{X} \Rightarrow \sum_{e}\left(w_{e}^{X} \mathbf{B}_{e j}^{X} \cdot \boldsymbol{\sigma}_{e}^{X}-\mathbf{f}_{j}^{X}\right)=0 \quad \forall j, X
\end{gathered}
$$

In the above equations, $S_{i}^{X}=\{e$ such that $s(X, e)=i\}$. Equation (6) simply states that each material strain $\boldsymbol{\epsilon}_{i}^{*}$ is a weighted average of the mechanical strains at quadrature points assigned to this specific material strain. Similarly, Equation (7) states that each material stress $\boldsymbol{\sigma}_{i}^{*}$ is a weighted average of the mechanical stresses at quadrature points assigned to this specific material stress. Combining Equations (7), (8) and (9) yields the following linear system, solved to simultaneously determine $\boldsymbol{\sigma}_{i}^{*}$ and $\boldsymbol{\sigma}_{e}^{X}$ (through $\boldsymbol{\eta}_{j}^{X}$ ):

$$
\sum_{k} \sum_{e} w_{e}^{X} \mathbf{B}_{e j}^{X}: \mathbb{C}_{e}^{X}: \mathbf{B}_{e k}^{X} \boldsymbol{\eta}_{k}^{X}+\sum_{e} w_{e}^{X} \mathbf{B}_{e j}^{X^{T}} \boldsymbol{\sigma}_{s(e, X)}^{*}=\mathbf{f}_{j}^{X} \forall j, X
$$

and

$$
\sum_{e \in S_{i}^{X}} \sum_{X} \sum_{j} w_{e}^{X} \mathbf{B}_{e j}^{X} \boldsymbol{\eta}_{j}^{X}=0 \quad \forall i
$$

The following algorithm is suggested for computing $\left(\boldsymbol{\epsilon}_{i}^{*}, \boldsymbol{\sigma}_{i}^{*}\right)_{i},\left(\boldsymbol{\sigma}_{e}^{X}\right)_{e, X}$ and $s:$

1. simultaneously initialize $\left(\boldsymbol{\epsilon}_{i}^{*}\right)_{i}$ and $s$ by a k-means algorithm on $\left(\boldsymbol{\epsilon}_{e}^{X}\right)_{e, X}$,

2. simultaneously compute $\left(\boldsymbol{\sigma}_{i}^{*}\right)_{i}$ and $\left(\boldsymbol{\eta}_{j}^{X}\right)_{j, X}$ from Equations (10-11),

3. update the value of $\left(\boldsymbol{\sigma}_{e}^{X}\right)_{e, X}$ using Equation (8), 
4. compute a new state mapping $s$ with:

$$
s=\arg \min _{s} \sum_{X} \sum_{e} w_{e}^{X}\left\|\left(\boldsymbol{\epsilon}_{e}^{X}-\boldsymbol{\epsilon}_{s(e, X)}^{*}, \boldsymbol{\sigma}_{e}^{X}-\boldsymbol{\sigma}_{s(e, X)}^{*}\right)\right\|_{\mathbb{C}_{e}}^{2}
$$

5. update $\left(\boldsymbol{\epsilon}_{i}^{*}\right)_{i}$ from Equation (6),

6. iterate steps 2-5 until convergence of $s$.

The DDI problem is mathematically a Mixed-Integer Quadratic Programming (MIQP) problem which is here solved heuristically. It is easy to observe that the cost function (3) decreases at each iteration, but there is no guarantee of convergence towards a global minimum. Following the work of Kanno [9], it is however reasonnable to suggest that the globally optimum solution of DDI can be achieved using standard Mixed Integer Programming methods such as branch and bound approaches. The computational cost of theses methods is however such that they cannot be applied here.

\section{Applications for quasi-static and dynamic problems}

\subsection{Quasi-static non-linear elasticity}

The method proposed in the previous section is applied to manufactured data. The problem consists in the identification of the mechanical response of a non-linear incompressible material under plane stress assumption. A 2D finite element mesh with 4111 nodes and 7764 triangular elements is considered. It is subjected to $N^{X}=40$ increasing traction steps in the $y$ direction. The mesh as well as a representative deformed configuration are 
depicted in Figure 1. The constitutive equation used in the FE simulations to generate the data is of the form:

$$
\begin{aligned}
& \boldsymbol{\sigma}=G\left(\boldsymbol{\epsilon}+\alpha \boldsymbol{\epsilon}^{3}\right)-p \mathbf{I} \\
& p=-\left(\epsilon_{x x}+\epsilon_{y y}\right)-\alpha\left(\epsilon_{x x}+\epsilon_{y y}\right)^{3} .
\end{aligned}
$$

where $G$ and $\alpha$ are the material parameters chosen as $G=1 \mathrm{MPa}$ and $\alpha=5$. We consider a tensor $\mathbb{C}_{e}$ corresponding to the linearization, i.e. $\alpha=0$, of the above equation with $G=100 \mathrm{MPa}$. The initialization of $s$ and $\left(\boldsymbol{\epsilon}_{i}^{*}\right)_{i}$ is obtained by applying a k-means algorithm to the set of $\left(\boldsymbol{\epsilon}_{e}^{X}\right)_{e, X}$. About 70 DDI iterations are necessary to converge for $N^{*}=15000$. This particular choice of parameters $\left(\mathbb{C}_{e}\right.$ and $\left.N^{*}\right)$ is guided by the preliminary parametric study of the method [8].

Since the "true" constitutive equation used to generate the data is isotropic, we first explore the misalignment between the identified material strain and stress. To this end, for each material state $\left(\boldsymbol{\epsilon}_{i}^{*}, \boldsymbol{\sigma}_{i}^{*}\right)$, the angle $\theta_{i}$ between the dominant eigenvectors of the strain and stress tensors is computed. The distribution of these misalignment angles is shown in Figure 2. As expected the misalignment is small and $80 \%$ of all material states have less than $0.5^{\circ}$ misalignment.

In Figure 3, we present the first eigenvalue of the material stress tensor $\left(\sigma_{I}^{*}\right)$ as a function of the eigenvalues of the corresponding material strain $\left(\epsilon_{I}^{*}, \epsilon_{I I}^{*}\right)$. All points fall very close to the surface built from the constitutive 
equation used to generate the input data:

$$
\frac{\sigma_{I}}{G}=\epsilon_{I}+\alpha \epsilon_{I}^{3}+\left(\epsilon_{I}+\epsilon_{I I}\right)+\alpha\left(\epsilon_{I}+\epsilon_{I I}\right)^{3} .
$$

The distribution of material strain in the $\left(\epsilon_{I}^{*}, \epsilon_{I I}^{*}\right)$ plane provides an importance sampling of the relevant strain states in the dataset. In particular one can observe the lack of data for equibiaxial deformation (i.e. $\left.\epsilon_{I}^{*}=\epsilon_{I I}^{*}\right)$.

The DDI algorithm provides the material states $\left(\boldsymbol{\epsilon}_{i}^{*}, \boldsymbol{\sigma}_{i}^{*}\right)_{i}$ database but also predicts the mechanical stresses $\left(\boldsymbol{\sigma}_{e}^{X}\right)_{e, X}$ of all snapshots. Figure 4 (left) presents the predicted von Mises stress for one of the snapshots, and Fig. 4 (middle) shows the relative error. The prediction is accurate to less than $5 \%$ in $95 \%$ of all elements, confirming the accuracy of the DDI method. For the whole collection of snapshots, the mean relative error in von Mises stress is $2.1 \%$.

\subsection{Wave propagation in linear elasticity}

Section 3.1 illustrates a quasi-static problem and consequently all nodal forces $\left(\mathbf{f}_{j}^{X}\right)_{j, X}$ appearing in the right-hand side of Equation (10) are zero excepted for the nodal forces corresponding to nodes subjected to Dirichlet Boundary conditions.

In this section we investigate the case of wave propagation, where the nodal forces $\left(\mathbf{f}_{j}^{X}\right)_{j, X}$ are inertial. Note that transient data-driven computational analysis have been proposed in [10]. Again, the problem consists in the identification of the mechanical response of an elastic material with 
the plane stress assumption but here, the successive snapshots used for the identification represent the time evolution of a pre-deformed specimen that is suddenly released. Figure 5 shows the initial configuration of the specimen and some representative configurations following the release of the upper boundary. The FE mesh consists of 9200 triangular elements and 4866 nodes. To generate the data, time integration is performed using a standard Newmark method. In this example a linear elastic constitutive model $(E=1 \mathrm{MPa}, \nu=0.3)$ has been used for the sake of simplicity. Nodal accelerations are re-computed a posteriori using centered finite difference approximation while the nodal forces are obtained by multiplying the mass matrix of the FE mesh with the vector of nodal accelerations. The parameters of the DDI algorithm are chosen as follows:

- the number of material states $N^{*}$ is selected to be approximately 0.05 times the number of mechanical states,

- the pseudo-elasticity tensor $\mathbb{C}_{e}$ is built using a modulus $E^{*}=100 \mathrm{MPa}$ and an arbitrary Poisson ratio $\nu^{*}=0.45$.

Convergence is reached after 89 iterations.

Figure 6 compares, for all snapshots, the identified mechanical stress with the actual true stress values computed from the underlying constitutive model. We observe that the predictions are globally very good, excepted for some values of $\sigma_{x x}$. This can actually be expected as the specimen was initially stretched mostly along the $y$-axis yielding less rich and intense data along $x$. Figure 7 shows the predicted mechanical stress $\sigma_{x y}$ along with the 
error magnitude for a particular snapshot. As expected from Fig. 6, the error levels are negligible compared to the actual stress value. Using the computed database of material states $\left(\boldsymbol{\epsilon}^{*}, \boldsymbol{\sigma}^{*}\right)$, the fit of a linear elastic law yields back the parameters of the linear constitutive model used to generate the data: $E_{\mathrm{id}}=1.01 \mathrm{MPa}$ and $\nu_{\mathrm{id}}=0.299$.

\section{Model order and mixed reduction}

In this section, an alternative resolution strategy is proposed. The main idea is that from a FE simulation of the experiment and a constitutive law obtained either from homogeneous stress experiments or a FEMU technique, a first guess (referred to as initial latter on) of the stress field (satisfying balance equations and balancing the external load) can be obtained. The strain field obtained from this simulation does not correspond to the actual measured strain. The DDI strategy proposed above is derived in order to obtain a non-parametric correction of the first guess of the stress field. This first guess may be correct in zones where the material state (stress, strain) fields remain homogeneous/simple and thus accurately predicted by the constitutive equation. Consequently, the correction field is searched for locally in a reduced domain (RD).

\subsection{Formulation}

For solving the problem stated in Section 2 locally, one needs to convert

the initial stress field $\tilde{\boldsymbol{\sigma}}_{e}^{X}$ to Neumann conditions on the boundary between 
the RD and the rest of the structure. Alternatively, one can search for a correction to the initial stress field. The stress field is written as

$$
\boldsymbol{\sigma}_{e}^{X}=\tilde{\boldsymbol{\sigma}}_{e}^{X}+\overline{\boldsymbol{\sigma}}_{e}^{X}
$$

where $\overline{\boldsymbol{\sigma}}_{e}^{X}$ is the self-balanced correction to the initial stress $\tilde{\boldsymbol{\sigma}}_{e}^{X}$. This correction being self-balanced, it is relevant to search it as part of a reduced space of admissible stress fields that can be parametrized (decomposed on a reduced basis). Suppose that a basis $\left(\mathbf{L}_{e}^{j}\right)_{j=1 . . N_{s}}$ of self-balanced stress fields is known, then one obtains

$$
\boldsymbol{\sigma}_{e}^{X}=\tilde{\boldsymbol{\sigma}}_{e}^{X}+\mathbf{L}_{e}^{j} \mathbf{P}_{j}^{X}
$$

where $\left(\mathbf{P}_{j}^{X}\right)_{j=1 . . N_{s}}$ are the parameters of the reduced basis for the snapshot $X$. Appendix 6 proposes a procedure to generate such a basis of self-balanced stress fields. It simply consists in a singular value decomposition of the linear operator allowing to compute the finite element internal force vector from a given stress field. The total strain associated to these correction fields vanishes, which is to be related to the non-uniqueness of the solution of the balance equation on its own. This means that the effect of this correction cannot be observed. The consistency of the corrected stress field with respect to the searched material states database is used to restore the well-posed nature of the problem. The optimization problem written in Equation (2) is recast as

$$
\text { solution }=\arg \min _{\mathbf{P}_{j}^{X}, \boldsymbol{\epsilon}_{i}^{*}, \boldsymbol{\sigma}_{i}^{*}, s} \mathcal{E}\left(\tilde{\boldsymbol{\sigma}}_{e}^{X}+\mathbf{L}_{e}^{j} \mathbf{P}_{j}^{X}, \boldsymbol{\epsilon}_{i}^{*}, \boldsymbol{\sigma}_{i}^{*}, s(e, X)\right) \text {. }
$$


The basis used to decompose the stress correction being self-balanced and the initial stress field balancing the external load, this optimization problem is solved with no additional constraint. Then, taking all possible variations yields the following set of equations:

$$
\begin{gathered}
\delta \boldsymbol{\epsilon}_{i}^{*} \Rightarrow \sum_{X} \sum_{e \in S_{i}^{X}} w_{e}^{X} \mathbb{C}_{e}^{X}:\left(\boldsymbol{\epsilon}_{e}^{X}-\boldsymbol{\epsilon}_{s(e, X)}^{*}\right)=0 \quad \forall i \\
\delta \boldsymbol{\sigma}_{i}^{*} \Rightarrow \sum_{X} \sum_{e \in S_{i}^{X}} w_{e}^{X}\left(\mathbb{C}_{e}^{X}\right)^{-1}:\left(\boldsymbol{\sigma}_{e}^{X}-\boldsymbol{\sigma}_{s(e, X)}^{*}\right)=0 \quad \forall i \\
\delta \mathbf{P}_{j}^{X} \Rightarrow \sum_{e} \sum_{k}\left(w_{e}^{X} \mathbf{L}_{e}^{k^{T}} \cdot\left(\mathbf{L}_{e}^{j} \mathbf{P}_{j}^{X}+\tilde{\boldsymbol{\sigma}}_{e}^{X}-\boldsymbol{\sigma}_{s(e, X)}^{*}\right)=0 \quad \forall j, X .\right.
\end{gathered}
$$

The interpretation of these equations is exactly the same as previously. The following algorithm is suggested for computing $\left(\boldsymbol{\epsilon}_{i}^{*}, \boldsymbol{\sigma}_{i}^{*}\right)_{i},\left(\mathbf{P}_{j}^{X}\right)_{j, X}$ and $s$ :

1. simultaneously initialize $\left(\boldsymbol{\epsilon}_{i}^{*}, \boldsymbol{\sigma}_{i}^{*}\right)_{i}$ and $s$ by a k-means algorithm on

$$
\left(\boldsymbol{\epsilon}_{e}^{X}, \tilde{\boldsymbol{\sigma}}_{e}^{X}\right)_{e, X}
$$

2. compute $\left(\mathbf{P}_{j}^{X}\right)_{j, X}$ from Equation (21),

3. compute $\left(\boldsymbol{\sigma}_{i}^{*}\right)_{i}$ from Equation (20),

4. compute a new state mapping $s$ with:

$$
s=\arg \min _{s} \sum_{X} \sum_{e} w_{e}^{X}\left\|\left(\boldsymbol{\epsilon}_{e}^{X}-\boldsymbol{\epsilon}_{s(e, X)}^{*}, \tilde{\boldsymbol{\sigma}}_{e}^{X}+\mathbf{L}_{e}^{j} \mathbf{P}_{j}^{X}-\boldsymbol{\sigma}_{s(e, X)}^{*}\right)\right\|_{\mathbb{C}_{e}}^{2},
$$

5. update $\left(\epsilon_{i}^{*}\right)_{i}$ from Equation (19),

6. iterate steps 2-5 until convergence of $s$.

\subsection{Results}

A rectangular sample of $100 \times 20 \mathrm{~mm}^{2}$ with a hole of diameter $5 \mathrm{~mm}$ is considered. It is assigned an elasto-plastic behaviour with linear isotropic hardening. The Young's modulus is $168 \mathrm{GPa}$, the initial yield stress $284 \mathrm{MPa}$ and 
the hardening coefficient $1.48 \mathrm{GPa}$. The Poisson's ratio is 0.25 . To generate strain data, a numerical simulation is run under prescribed displacement at the left and right ends of the sample with a mesh similar to the one shown in Figure 8(a). A 2D plane stress formulation is adopted. The macroscopic response obtained in the simulation is illustrated in Figure 9.25 steps are performed.

The initial stress field is obtained from a purely elastic simulation (balancing the external load) using the elastic tensor of the material. $\mathbb{C}_{e}$ is set equal to the actual elastic tensor. The Young modulus of the material being about 100 times higher than its hardening modulus, this choice is consistent with the recommendation in [8] when the focus is on the elasto-plastic response of the material. The $\mathrm{RD}$ on which the analysis is performed consists in the elements that have their total strain norm exceeding $0.2 \%$. Two layers of elements are added to this RD as depicted in Figure 10. The RD finally contains about 2000 elements. In practice, the RD should be defined to ensure that plasticity remains embedded within the $\mathrm{RD}$, meaning that along the RD boundary $\bar{\sigma}^{e} n=0$ is a valid assumption. The entire basis of self-balanced stress field computed over this RD is used for the DDI analysis which involves 800 material points in the database for analyzing the last 5 snapshots. To illustrate the potential of using model order reduction, the normalized amplitude of the self-balanced modes used to correct the initial stress field at the last snapshot is plotted in Figure 14. It is clearly shown that the correction obtained with the full basis could have been ob- 
tained with a sufficiently high level of accuracy (compared the measurement uncertainty) with half the number of modes.

After the analysis is run, the actual elastic tensor is used to reconstruct the elastic strain (including the out-of-plane component assuming plane stress) from the stress and then the plastic strain (including the out-of-plane component assuming plastic incompressibility). In Figure 11, the von Mises norm of the plastic strain is plotted for the last snapshot. At this last step, the von Mises norm of the stress field is presented in Figure 12(b). It is to be compared to the initial elastic stress in Figure 12(a). This correction is consistent with the actual strain shown in Figure 8(b) which is very close to the plastic strain as expected for such an elasto-plastic material. To further check for the consistency of identified stress field and material state database, a von Mises stress v.s. plastic strain plot gathering the data from all the snapshots is proposed in Figure 13; the actual constitutive law of the material used to generate the strain and force data is also plotted. A good agreement with both mechanical and material states is obtained.

\section{Summary and concluding remarks}

In this paper, it is shown that an inverse problem for the data-driven computational mechanics approach can be formulated and solved efficiently. It allows for obtaining, with no assumption on the relationship between stress and strain, a database of admissible material state. This database is a sampling of the full-field of strain and reconstructed stress that fulfill all the 
governing equations of a mechanical problem: balance of momentum, compatibility, and boundary conditions. The resolution of the inverse problem is performed either globally or on local patches where the actual behaviour of the material is supposed to differ from an initial guess. The only data that is required for the analysis are: the geometry of the tested sample, and both measured displacement fields and corresponding load measurements.

Through the three examples presented in the paper, the ability of the method to handle with different type of non-linearities and loading conditions (dynamic being considering as a volume force) is illustrated. The main limitations of the proposed approach are:

- in its present formulation the method is restricted to material behaviors for which the stress is a function of the strain, such as (non-linear) elasticity and monotonous radial loading in the elasto-plastic case;

- due to the non interpolating / extrapolating nature of the method, rich experiments involving heterogeneous deformations are required.

This will be the purpose of future investigations including the analysis of real complex experimental data sets.

\section{Acknowledgments}

The support of Région Pays de la Loire and Nantes Métropole through grant Connect Talent IDS is gratefully acknowledged. 


\section{Appendix: Self-balanced stress field computation}

The elaboration of the basis is performed using a singular value decomposition of the elementary internal force vector. Using the notations introduced in the paper, any elementary stress field having one component $c$ activated in one single element $k$ gives rise to finite element internal forces

$$
\mathbf{f}_{j}^{k, c}=\sum_{e} w_{e} \mathbf{B}_{e j}{ }^{T} \cdot \boldsymbol{\sigma}_{k}^{c} \quad \forall j, k, c .
$$

A singular value decomposition of this set of internal force vector is carried out to obtain the combination of elementary stress $\boldsymbol{\sigma}^{s}$ giving rise to vanishing internal force vector. If $N^{n}$ denotes the number of nodes in the finite element mesh, the internal force vector has $2 N^{n}$ components in $2 \mathrm{D}$. The number of independent elementary stress being $3 N^{e}\left(N^{e}\right.$ being the number of quadrature points), the number of self-balanced stress fields is $3 N^{e}-2 N^{n}$ in $2 \mathrm{D} . \mathbf{L}_{e}^{s}$ is simply computed as the singular vectors of the matrix gathering all $\mathbf{f}_{j}^{k, c}$, corresponding the $3 N^{e}-2 N^{n}$ smallest singular values.

\section{References}

1. MA Sutton, JJ Orteu, and H Schreier. Image correlation for shape, motion and

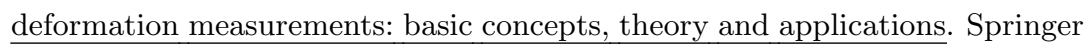
Science \& Business Media, 2009.

2. D. Lecompte, A. Smits, H. Sol, J. Vantomme, and D. Van Hemelrijck. Mixed numerical-experimental technique for orthotropic parameter identification using biaxial tensile tests on cruciform specimens. International Journal of Solids and Structures, 44(5):1643-1656, 2007. 
3. J. Réthoré. A fully integrated noise robust strategy for the identification of constitutive laws from digital images. International Journal for Numerical Methods in Engineering, 84:631-660, 2010.

4. J. Réthoré, Muhibullah, T. Elguedj, M. Coret, P. Chaudet, and A. Combescure. Robust identification of elasto-plastic constitutive law parameters from digital images using 3d kinematics. International Journal of Solids and Structures, 50(1):73-85, 2013.

5. F Hild and S Roux. Digital image correlation: from displacement measurement to identification of elastic properties-a review. Strain, 42(2):69-80, 2006.

6. S Avril, M Bonnet, AS Bretelle, M Grédiac, F Hild, P Ienny, F Latourte, D Lemosse, S Pagano, E Pagnacco, et al. Overview of identification methods of mechanical parameters based on full-field measurements. Experimental Mechanics, 48(4):381, 2008.

7. T Kirchdoerfer and M Ortiz. Data-driven computational mechanics. Comput. Methods Appl. Mech. Engrg., 304:81-101, June 2016.

8. A. Leygue, M. Coret, J. Réthoré, L. Stainier, and E. Verron. Data-based derivation of material response. Computer Methods in Applied Mechanics and Engineering, 331:184-196, 2018.

9. Y Kanno. Mixed-integer programming formulation of a data-driven solver in computational elasticity. Optim. Lett., February 2019.

10. $\mathrm{T}$ Kirchdoerfer and $\mathrm{M}$ Ortiz. Data-driven computing in dynamics.

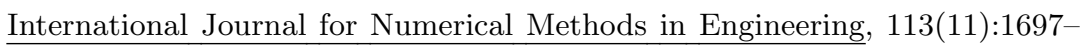
$1710,2018$. 

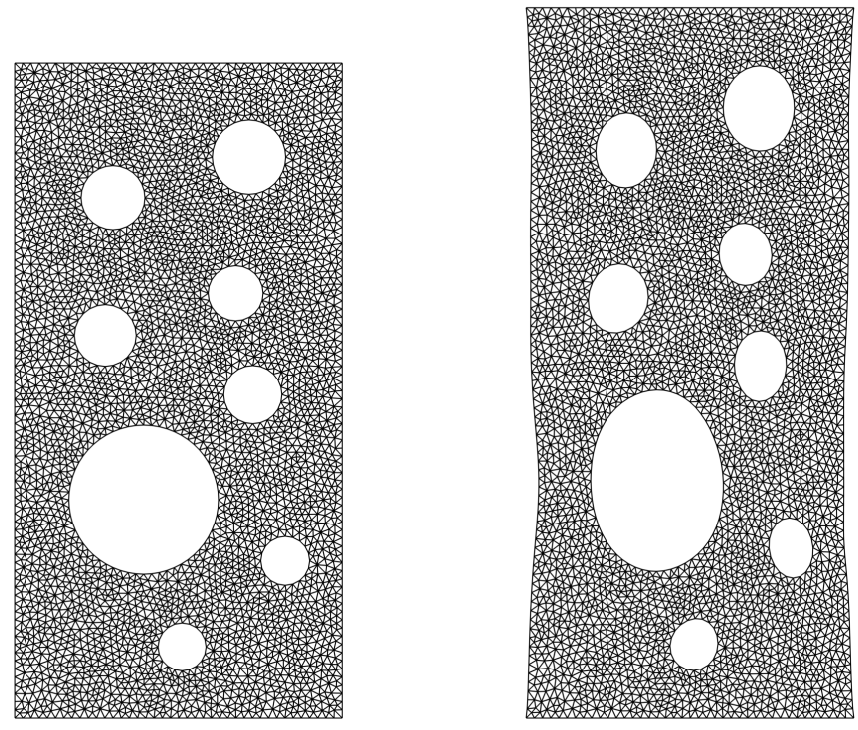

Fig. 1 Computational mesh of 4111 nodes and 7764 triangular elements (left) and typical deformed configuration (right). 


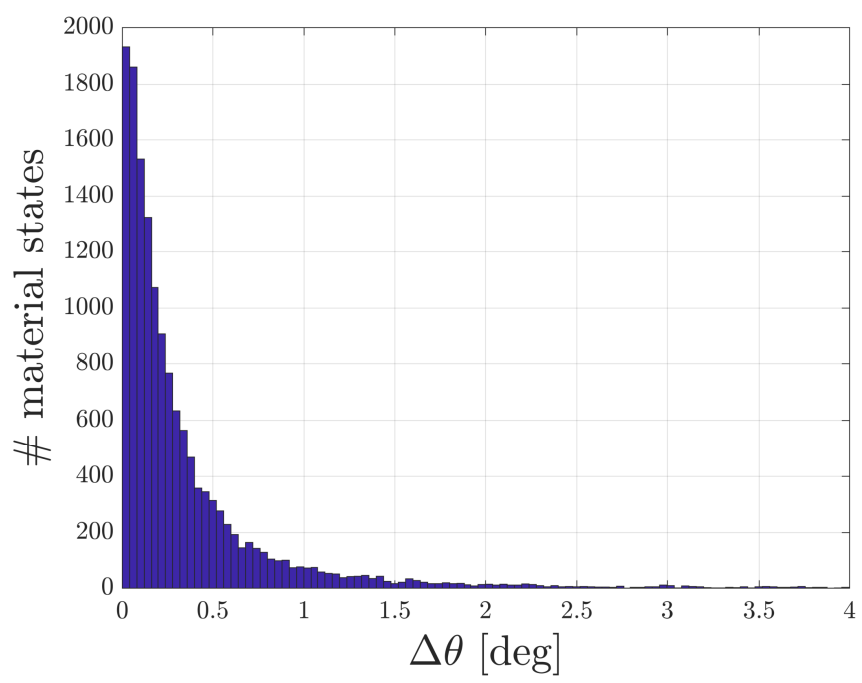

Fig. 2 Distribution of misalignment angle between the material strain $\epsilon^{*}$ and material stress, $\boldsymbol{\sigma}^{*}$.

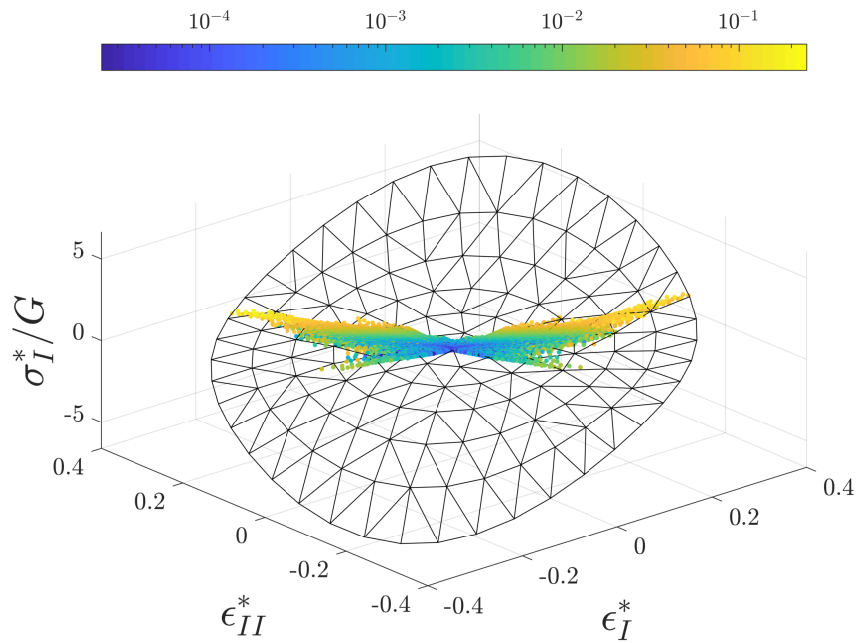

Fig. 3 Largest eigenvalue of the material stress tensor as a function of the eigenvalues of the material strain tensor. The symbols are computed from the identified material states $\left(\boldsymbol{\epsilon}^{*}, \boldsymbol{\sigma}^{*}\right)$ and the surface from Equation (15). The color denotes the vertical distance between the symbols and the analytical surface.. 

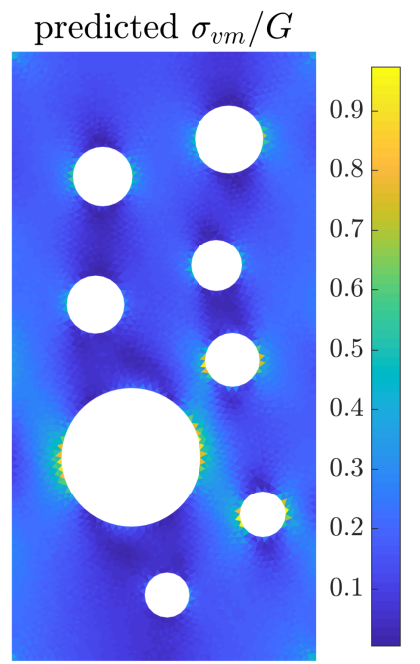

relative error in $\sigma_{v m}$

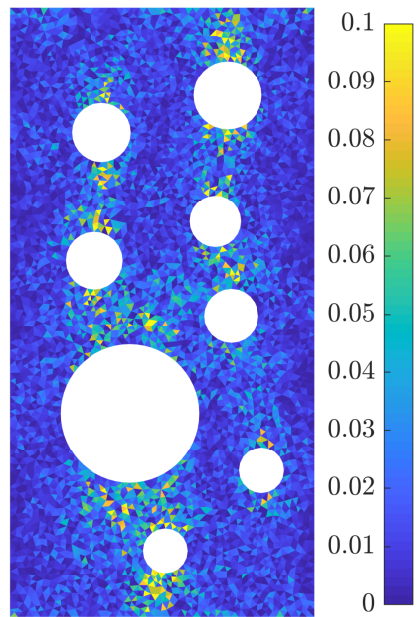

Fig. 4 Predicted von Mises mechanical stress for one of the snapshots (left) and relative error (right) .
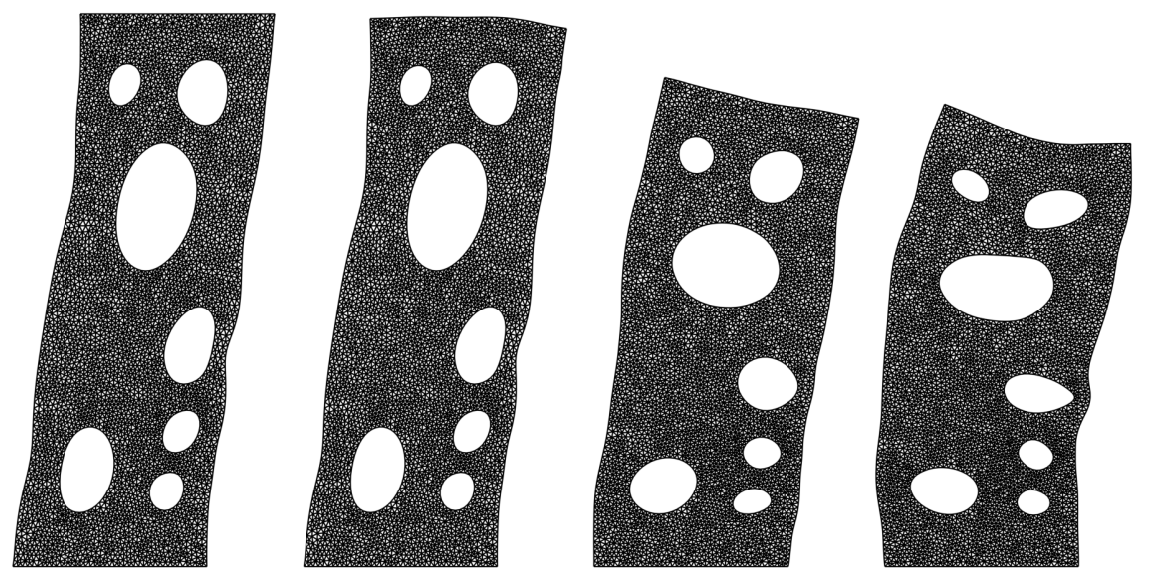

Fig. 5 Initial configuration (left) and some representative snapshot configurations 

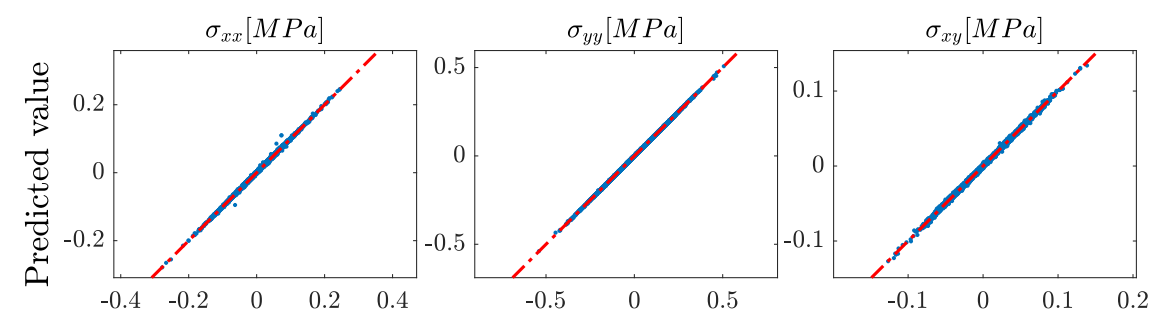

Exact value

Fig. 6 Predicted mechanical stress components [MPa] vs. "true" FE stress components $[\mathrm{MPa}]$.

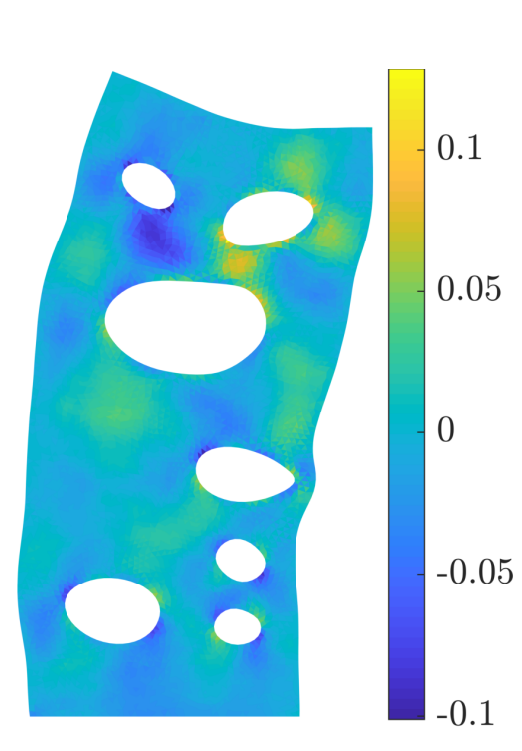

identified $\sigma_{x y}[\mathrm{MPa}]$

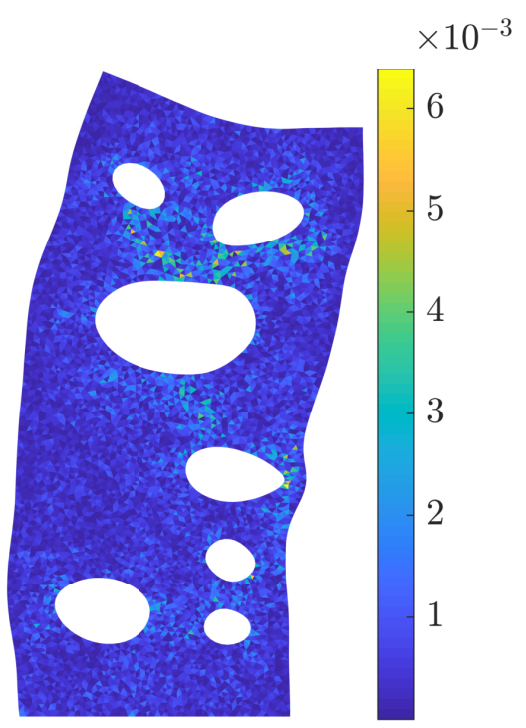

error magnitude for $\sigma_{x y}[\mathrm{MPa}]$

Fig. 7 Identified stress for one of the snapshots (left) and error magnitude (right). 


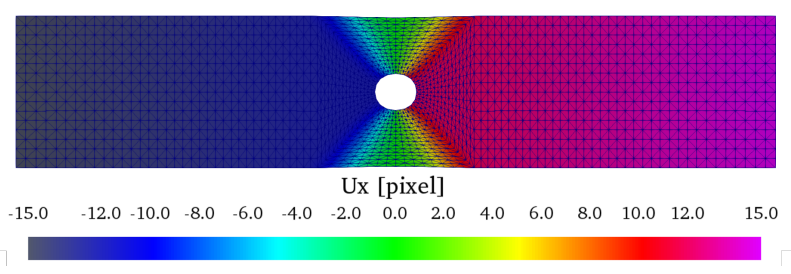

(a)

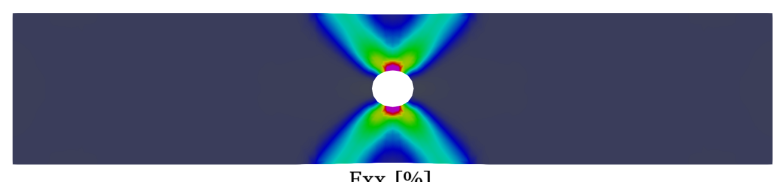

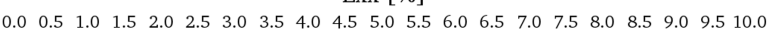

(b)

Fig. 8 Mesh used for the elasto-plastic analysis and horizontal displacement and strain for the last snapshot.

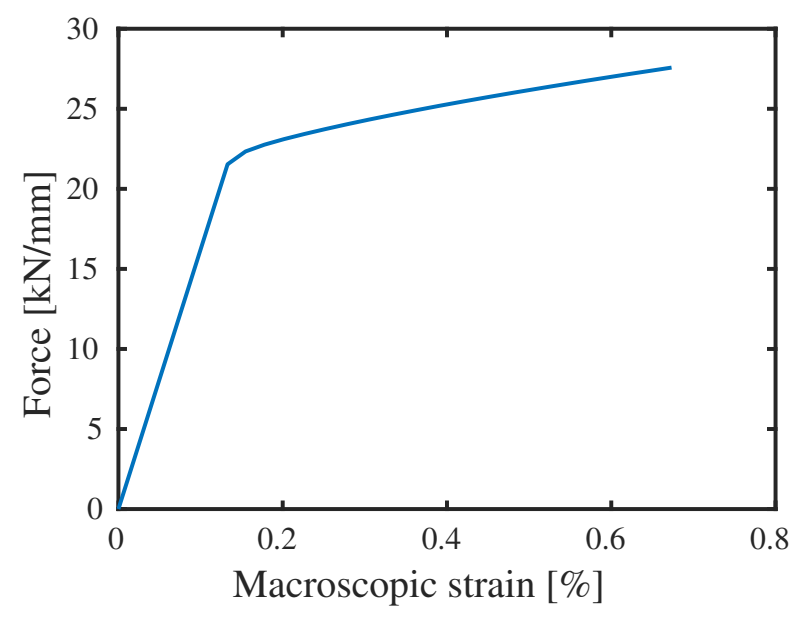

Fig. 9 Macroscopic response of the holed plate with an elasto-plastic behavior. 


\section{$\bullet$}

Fig. 10 The pink zone is used as a reduced domain for the DDI.

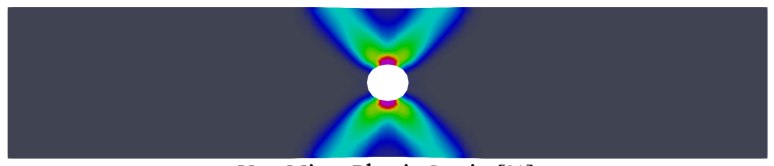

Von Mises Plastic Strain [\%]

$\begin{array}{lllllllllllllllllllll}0.0 & 0.5 & 1.0 & 1.5 & 2.0 & 2.5 & 3.0 & 3.5 & 4.0 & 4.5 & 5.0 & 5.5 & 6.0 & 6.5 & 7.0 & 7.5 & 8.0 & 8.5 & 9.0 & 9.5 & 10.0\end{array}$

Fig. 11 von Mises norm of the plastic strain for the last snapshot.

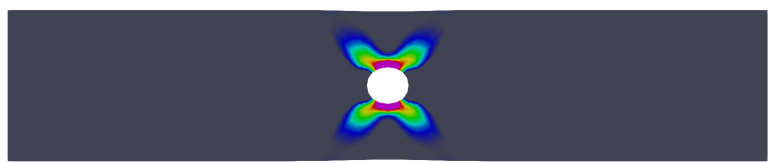

Von Mises Stress [MPa]

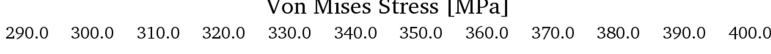

(a)

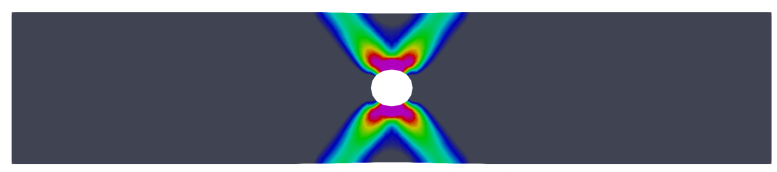

Von Mises Stress $[\mathrm{MPa}]$

$\begin{array}{llllllllllll}290.0 & 300.0 & 310.0 & 320.0 & 330.0 & 340.0 & 350.0 & 360.0 & 370.0 & 380.0 & 390.0 & 400.0\end{array}$

(b)

Fig. 12 Comparison of the von Mises norm of initial (elastic) stress (a), recon-

structed stress (b) for the last snapshot. 


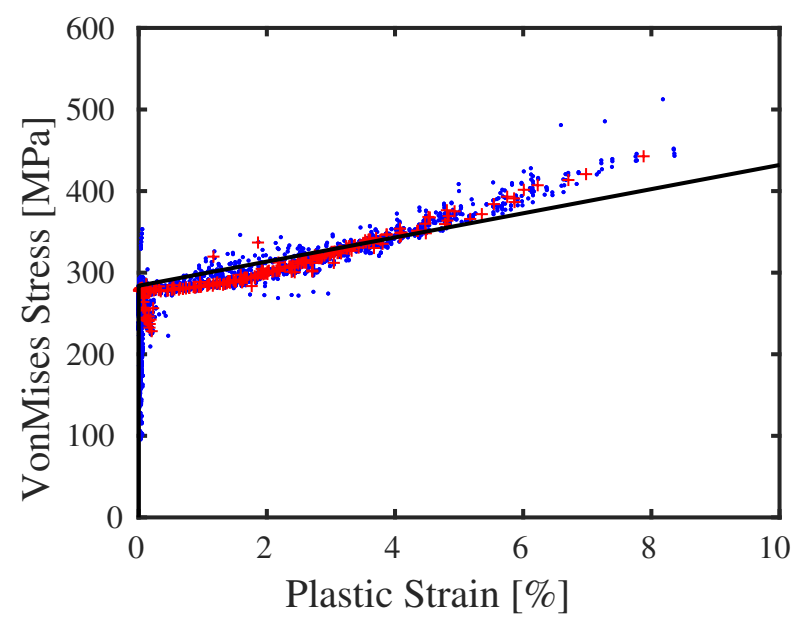

Fig. 13 Stress v.s. plastic strain representation of the reconstructed state fields. The black line corresponds to the constitutive law used to generate the displacement data. The blue points represent the mechanical states and the red points the material state database.

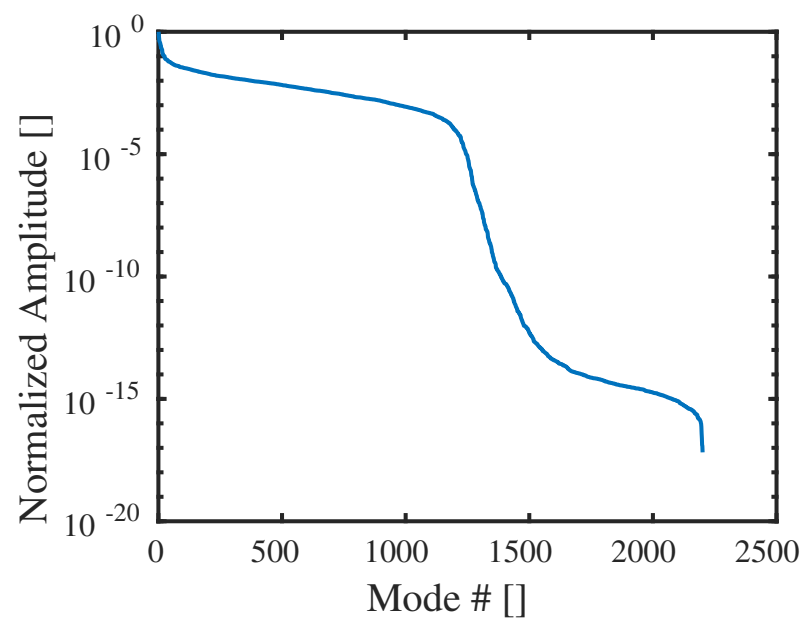

Fig. 14 Normalized amplitude (for the last snapshot) of the self-balanced modes used to correct the initial stress field. 